\title{
Bestimmung der „Leucinaminopeptidase“ des Rattenharnes unter Verwendung von L-Leucyl- $\beta$-naphthylamin und L-Leucyl-p-nitroanilid
}

\author{
Von W. RAAB \\ Aus dem Universitätsinstitut für Medizinische Chemie der Universität Wien (Vorstand: Prof. Dr. F. Seelicb)
}

(Eingegangen am 27. September 1965)

\begin{abstract}
Es wurde geprüft, ob das neuerdings zur Bestimmung der sogenannten Leucinaminopeptidase („LAP“) empfohlene Substrat L-Leucyl-p-nitroanilid für Untersuchungen der renalen LAP geeignet ist. Nach Bestimmung der linearen Korrelation zwischen Abspaltung von $\mathrm{p}$-Nitroanilin und $\beta$-Naphthylamin (aus L-Leucyl- $\beta$-naphthylamin nach der alten Methode) durch die LAP des Rattenharnes unter normalen Bedingungen (200 Ratten) wurden die Untersuchungen auch auf Schocktiere ausgedehnt, bei denen eine starke Vermehrung der LAP-Ausscheidung vorlag (100 Ratten). Auch hier erwies sich das neue Substrat (LEUPA) als ebenso geeignet für die Bestimmung der LAP des Harnes wie das bisher verwendete LEUNA.

After some introductory remarks on the specifity of leucine-aminopeptidase („LAP“) a simplified method using leucine-p-nitroaniline (LEUPA) instead of leucine- $\beta$-naphthyl-amide (LEUNA) for the determination of urinary LAP is reported. Comparative investigations with both substrates revealed a linear correlation between the splitting of LEUNA and LEUPA, not only under normal conditions (200 rats) but also after the experimental increase of urinary LAP-excretion (100 rats). The good results recommend the simplified method for determinations of urinary LAP.
\end{abstract}

Definitionsgemäß versteht man unter Leucinaminopeptidasen „LAP“ eine bestimmte Gruppe von Exopeptidasen, die am Ende von Peptidketten Leucylreste abzuspalten vermögen. L-Leucylamid stellt das spezifische Substrat für echte LAP aus Schweinenieren (1) oder Rinderaugenlinsen (2) dar. Bei den üblicherweise als „Leucinaminopeptidase“ bezeichneten Enzymen handelt es sich eigentlich um Aminosäurearylamidasen (3). Das von GREEN und Mitarbeitern (4) vorgeschlagene Substrat L-Leucyl- $\beta$-naphthylamin („LEUNA“), das bis vor kurzem fast ausschließlich zur Bestimmung der LAP herangezogen wurde, kann auch durch andere Enzyme, zum Beispiel durch verschiedene Endopeptidasen (vgl.5) aufgespalten werden. Deshalb wurde für die Gesamtheit der mit diesem Substrat erfaßten Enzyme der Ausdruck "L-Leucyl- $\beta$-naphthylamin-Hydrolase" vorgeschlagen (6). Diese Bezeichnung hat sich jedoch nicht durchsetzen können; der Ausdruck „Leucinaminopeptidase“ wurde beibehalten, allerdings unter Vorsetzung des Wörtchens „sogenannt", um die mangelnde Spezifität der geübten Nachweismethode auszudrücken.

Die Bestimmung der LAP unter Verwendung von LEUNA als Substrat ist technisch nicht ganz einfach. 1962 haben Tuppy und Mitarbeiter (7) eine vereinfachte Methode zur Bestimmung der LAP angegeben, wobei LLeucyl-p-nitroanilid als Substrat eingeführt wurde. Bei dieser Methode entfällt die bisher notwendige Fällung und Abtrennung der Serumeiweißkörper. Außerdem kann das enzymatisch gebildete Nitroanilin direkt photometrisch bestimmt werden. Die bisher notwendige Kupplung des $\beta$-Naphthylamins mit Echtrot und die Extraktion des gebildeten Farbstoffes werden überflüssig. Aus letzteren Gründen erscheint die Verwendung von L-Leucyl-p-nitroanilid („LEUPA“) auch bei Bestimmung der LAP des Harnes von Vorteil.

Vergleichende Untersuchungen der LAP des Serums unter Verwendung von LEUNA und LEUPA ergaben eine direkte lineare Abhängigkeit der Abspaltung von
$\beta$-Naphthylamin bzw. von p-Nitroanilin (3). Über die LAP des Harnes, deren Bestimmung große Bedeutung für die Früherfassung von tubulären Funktionsstörungen der Niere zukommt (8), liegen derartige Vergleichsuntersuchungen noch nicht vor. Die Annahme, daß LAP des Serums und LAP des Harnes ein gleiches Verhalten zeigen müssen, erscheint nicht berechtigt. Biochemische Untersuchungen ergaben in manchen Punkten ein unterschiedliches Verhalten; so liegen zum Beispiel die pH-Optima verschieden (9). Wenn keine pathologischen Veränderungen (z. B. Proteinurie) vorliegen, besteht keinerlei Abhängigkeit zwischen LAPAktivität des Serums und LAP-Aktivität des Harnes. Unter normalen Bedingungen dürfte die LAP des Harnes ausschließlich auf die Zellmauserung von Nie-" renepithelien zurückgehen.

Diè LAP des Rattenharnes läßt unter normalen und pathologischen Bedingungen eine gute Vergleichbarkeit mit der LAP des menschlichen Harnes erkennen $(8,10)$. In den vorliegenden Untersuchungen wurde deshalb Harn von normalen Ratten und von Ratten mit experimentell gesteigerter LAP-Ausscheidung für vergleichende LAP-Bestimmungen unter Verwendung von LEUNA und LEUPA herangezogen.

\section{Methodik \\ Versucbstiere}

Männliche Ratten erhielten täglịch 10,0 g Wasser mit der Schlundsonde verfüttert um vergleichbare Harnmengen zu erzielen. Der Harn wurde in Stoffwechselkäfigen aufgefangen und in 24-Stunden-Portionen untersucht. Nach Bestimmung der Normalwerte bei 200 Tieren wurde bei 50 Tieren ein anaphylaktischer Schock (Sensibilisierung: Humanalbumin $+6 \times 10^{9}$ Keime einer PertussisVakzine, Einzelheiten siehe $(11,12)$ ) ausgelöst. Bei weiteren 50 Tieren erfolgte die Auslösung eines anaphylaktoiden Schocks durch intraperitoneale Injektion einer 
synthetischen, mastzelldegranulierenden Substanz. Verwendet wurde "Compound 48/80" (vgl. 13, 14) in einer Dosierung von $2 \mathrm{mg}$ pro Kilogramm Körpergewicht.

\section{Bestimmung der LAP unter Verwendung von L-Leucyl- $\beta$ - napbtbylamid. (LEUNA)}

$4 \mathrm{mg}$ LEUNA-HCl in $2,0 \mathrm{ml} 0,05 m$ Phosphatpuffer (pH $=7,2$ ) wurden mit $0,05 \mathrm{ml}$ frischem zentrifugiertem Harn 1 Stunde bei $37^{\circ}$ inkubiert. Die Beendigung der enzymatischen Reaktion erfolgte durch Zugabe von $1 \mathrm{ml}$ einer 60-proz. Perchlorsäure. Das gebildete $\beta$-Naphthylamin wurde durch Kupplung mit Echtrot $3 \mathrm{GL}$ in einen roten Azofarbstoff übergeführt. Der Farbstoff wurde mit Äthylacetat ausgeschüttelt, kurz zentrifugiert und bei $492 \mathrm{~m} \mu$ photometrisch bestimmt. Die Auswertung erfolgte anhand einer Eichkurve mit verschiedenen Mengen an $\beta$-Naphthylamin.

Gemäß der Empfehlung der IUB wird eine Einheit an LAP-Aktivität definiert als die Enzymaktivität von $1 \mathrm{~m} l$ Harn, die unter den beschriebenen Versuchsbedingungen $1 \mu \mathrm{g} \beta$-Naphthylamin abspaltet.

\section{Bestimmung der LAP unter Veruendung von L-Leucyl-p- nitroanilid ( $L E U P A$ )}

$0,01 \mathrm{Mol}$ LEUPA wurden in $3,0 \mathrm{ml} 0,05 \mathrm{~m}$. Phosphatpuffer (pH $7,2)$ mit $0,1 \mathrm{ml}$ frischem, zentrifugiertem Harn 30 Minuten bei $25^{\circ}$ inkubiert. Das gebildete Spaltprodukt p-Nitroanilin kann direkt photometrisch bei $405 \mathrm{~m} \mu$ bestimmt werden, wobei die Messung vor Beginn der Inkubation als Bezugswert dient. Nach Multiplikation der Extinktionszunahme mit 0,108 erhält man den Wert der LAP-Einheiten (zumeist als. Millieinheiten $=m U$ anzugeben) pro $\mathrm{ml}$ Harn.

\section{Ergebnisse}

Bei jeder einzelnen Bestimmung war eine lineare Korrelation zwischen der Abspaltung von $\beta$-Naphthylamin und der Abspaltung von p-Nitroanilin zu beobachten. Die Umrechnung von mU LAP (gemessen bei Verwendung von LEUPA) in E LAP (gemessen bei Verwen- dung von LEUNA) kann durch Multiplikation mit dem Faktor 11 durchgeführt werden.

Die Ergebnisse finden sich in Tabelle 1 zusammengestellt. Die normale LAP-Aktivität im 24-Stunden-Harn beträgt $940 \pm 493 \mathrm{E}$ oder $85,5 \pm 41,6 \mathrm{mU}$. In der Tabelle 1 findet sich auch eine Zusammenstellung der gemessenen Aktivitäten pro $\mathrm{m} l$. Hierbei fällt eine größere Streuungsbreite der Werte auf; diese erklärt sich mit den mitunter stark wechselnden Harnmengen. Die durchschnittliche Harnausscheidung in 24 Stunden pro Ratte betrug $14 \mathrm{ml}$.

Die Auslösung eines anaphylaktischen oder anaphylaktoiden Schocks bewirkt eine statistisch signifikante Erhöhung der LAP-Ausscheidung im Harn. Die Zunahme war bei Bestimmung mit LEUNA gleichhoch wie bei Bestimmung mit LEUPA. Auch hier.fand sich bei jeder einzelnen Bestimmung die lineare Korrelation zwischen der Abspaltung von $\beta$-Naphthylamin und $\mathrm{p}$-Nitroanilin.

In Tabelle 2 wurde die prozentuale Zunahme der LAPAktivität im Rattenharn nach Auslösung eines anaphylaktischen und anaphylaktoiden Schocks zusammengestellt, wobei wieder eine Gegenüberstellung der Ergebnisse bei Anwendung von LEUNA und bei Anwendung von LEUPA erfolgte. Es fanden sich gleichhohe Aktivitätszunahmen, unabhängig davon ob ein anaphylaktischer oder anaphylaktoider Schock ausgelöst wurde und unabhängig vom verwendeten Substrat.

\section{Diskussion}

Schockreaktionen verschiedenster Art bewirken einen Blutdruckabfall und eine reflektorische Kontraktion der Nierengefäße (vgl. 3). Die hieraus resultierende Hypoxie verursacht eine Schädigung der hochempfindlichen Epi-

Tab. 1

Die LAP-Aktivität im Harn normaler Ratten und nach Auslösung eines Schocks

\begin{tabular}{cccc}
\hline Versuchstiere & Substrat & pro $\mathrm{ml}$ & Aktivität \\
& & im 24-Stdn.-Harn \\
\hline Normale Ratten (200 Tiere) & LEUNA & $71 \pm 40 \mathrm{E}$ & $940 \pm 493 \mathrm{E}$ \\
& LEUPA & $6,1 \pm 3,1 \mathrm{mU}$ & $85,5 \pm 41,6 \mathrm{mU}$ \\
Nach anaphylaktoidem Schock & LEUNA & $411 \pm 281 \mathrm{E}$ & $6002 \pm 1020 \mathrm{E}$ \\
(50 Tiere) & LEUPA & $36,3 \pm 24,1 \mathrm{mU}$ & $589,0 \pm 122,0 \mathrm{mU}$ \\
Nach anaphylaktischem Schock & LEUNA & $412 \pm 206 \mathrm{E}$ & $6115 \pm 428 \mathrm{E}$ \\
(50 Tiere) & LEUPA & $35,2 \pm 18,3 \mathrm{mU}$ & $540,1 \pm 111,3 \mathrm{mU}$ \\
\hline
\end{tabular}

Tab. 2

Prozentualer Anstieg der LAP-Aktivität im Rattenharn nach Auslösung eines Schocks

\begin{tabular}{|c|c|c|c|c|c|}
\hline \multirow{3}{*}{ Normalwerte } & \multirow{3}{*}{$\begin{array}{l}\text { Substrat } \\
\text { LEUNA } \\
\text { LEUPA }\end{array}$} & \multicolumn{4}{|c|}{ Aktivität } \\
\hline & & \multicolumn{2}{|c|}{$\begin{array}{l}\text { pro } \mathrm{ml} \\
\text { Anstieg auf }\end{array}$} & \multicolumn{2}{|c|}{$\begin{array}{l}\text { im 24-Stdn.-Harn } \\
\text { Anstieg auf }\end{array}$} \\
\hline & & $\begin{array}{l}71 \mathrm{E}(100 \%) \\
6 \mathrm{mU}(100 \%)\end{array}$ & - & $\begin{array}{l}940 \mathrm{E}(100 \%) \\
86 \mathrm{mU}(100 \%)\end{array}$ & $\overline{-}$ \\
\hline Anaphylaktoider Schock & $\begin{array}{l}\text { LEUNA } \\
\text { LEUPA }\end{array}$ & $\begin{array}{l}411 \mathrm{E} \\
36 \mathrm{mU}\end{array}$ & $\begin{array}{l}580 \% \\
600 \%\end{array}$ & $\begin{array}{l}6002 \mathrm{E} \\
589 \mathrm{mU}\end{array}$ & $\begin{array}{l}640 \% \\
680 \%\end{array}$ \\
\hline Anaphylaktischer Schock & $\begin{array}{l}\text { LEUNA } \\
\text { LEUPA }\end{array}$ & $\begin{array}{l}412 \mathrm{E} \\
35 \mathrm{mU}\end{array}$ & $\begin{array}{l}580 \% \\
580 \%\end{array}$ & $\begin{array}{l}6115 \mathrm{E} \\
540 \mathrm{mU}\end{array}$ & $\begin{array}{l}650 \% \\
630 \%\end{array}$ \\
\hline
\end{tabular}


thelzellen des proximalen Tubulus. Die in diesen Zellen enthaltenen Enzyme gelangen in den Harn. Normalerweise stammt die LAP-Aktivität des Harnes aus dem Prozeß der Zellmauserung an den Tubuli; nach Auslösung von Schockreaktionen wird nun das gleiche Enzym hochgradig vermehrt ausgeschieden. Zweifellos ist dieser Mechanismus am Zustandekommen der erhöhten proteolytischen Aktivität des Harnes nach Auslösung allergischer Reaktionen (15-19) mitbeteiligt. Darüber hinaus kann auch durch das bei allergischen Reaktionen aktivierte Plasmin (18) eine Aktivierung von Nierenpeptidasen erfolgen (20).
In den vorliegenden Versuchen ergab sich eine lineare Korrelation zwischen der Abspaltung von $\beta$-Naphthylamin und p-Nitroanilin durch die LAP des Harnes. Diese lineare Korrelation fand sich nicht nur unter normalen Bedingungen, sondern sie war auch bei pathologisch gesteigerter Ausscheidung von renaler LAP nachweisbar. L-Leucyl-p-Nitroanilid ist also zur Aktivitätsbestimmung der renalen LAP ebensogut geeignet wie L-Leucyl- $\beta$-Naphthylamin. Es gilt aber auch hier die in der Einleitung gemachte Einschränkung, daß keineswegs eine ausschließliche Bestimmung der echten Leucinaminopeptidase durchgeführt wird.

\title{
Literatur
}

1. Spackman, D. H., E. L. Smith und D. M. Brown, J. biol. Chemistry 212, 255 (1955). - 2. HaNson, H., D. Glaesser und H. KirschKe, Hoppe-Seylers' Z. physiol. Chem. 340, 107 (1965). 3. NAGEL, W., F. Willig und F. H. Schmidr, Klin. Wschr. 42, 447 (1964). - 4. Green, M. N., K.-C. Tsou, R. Bressler und A. M. Seligman, Arch. Biochemistry 57, 458 (1955). - 5. Steigleder, G.-K., Y. Kamei und R. Kudicke, Arch. klin. exp. Derm. 217, 417 (1963). - 6. O'Conell, W. und R. J. WinzleR, Cancer Res. 23, 78 (1963). - 7. Tuppy, H., U. Wiesbauer und E. WintersBerger, Hoppe-Seyler's Z. physiol. Chem. 329, 278 (1962). 8. BergmanN, H. und F. Scheler, Klin. Wschr. 42, 275 (1964). 9. Klaus, D., Ärztl. Forsch., Wörishofen 15, 548 (1961). - 10. BergmanN, H. und F. Truss, Med. Welt, 1964, 1760. - 11.
RAAB, W., Allergie und Asthma, im Druck. - 12. RAAB, W.: Renal enzyme excretion following anaphylactic shock Nature (London) im Druck. - 13. RAAB, W., Hautarzt 14, 241 (1963). 14. RAAB, W. und E. KAISER, Experientia, Basel, 21, 720 (1965). 15. Damganrd, E. und G. Ungar, Amer. J. Physiol. 171, 717 (1952). - 16. UNGAR, G., T. YAMURA, J. B. Isola und S. Kobrin, J. Exper. Med. 113, 359 (1961). - 17. KAISER, E., W. RAAB und W. LEPIER, Arch. klin. exp. Derm. 220, 321 (1964). - 18. RAAB, W. und E. KAISER, Klin. Wschr. 43, 345 (1965). - 19. RAAB, W. und E. Karser, Allergie und Asthma, 11, 37 (1965). - 20. INNERfield, I., R. Harvey, F. Luongo und E. Blincoe, Proc. Soc. exp. Biol. Med. 116, 573 (1964).

Dr. Wolfgang Raab

Universitäts-Institut für Med. Chemie, 1090 Wien (Osterreich), Währingerstraße 10

\section{Elektrophorese in horizontalem Polyacrylamidgel}

\section{Mitteilung: Einfluß verscbiedener Elektrophoresebedingungen auf die Auflösung von Humanserum}

\author{
Von O. ZwisLer und H. BIEL \\ Aus der Bebringwerke $\stackrel{A}{ } \bar{G}$, Marburg/Labn \\ (Eingegangen am 14. Juli 1965)
}

\begin{abstract}
Es wird eine mit einfachen Mitteln durchführbare Methode der Elektrophorese in horizontal gelagertem, nicht gekühltem Polyacrylamid-Gel beschrieben. Der Einfluß von verschiedenen Puffern, der Gelkonzentration und des Quervernetzungsgrades, der Laufstrecke und der Proteinkonzentration auf die elektrophoretische Trennung von Humanserum wurde untersucht. Humanserum wird in mindestens 20 unterscheidbare Komponenten zerlegt.

A method employing simple materials for electrophoresis in horizontally layered, non-cooled polyacrylamide gel is described. The influence of different buffers, gel concentration, degree of cross linkage, running distance and protein concentration on the electrophoretic separation of human serum were studied. Human serum was separated into at least 20 different components.
\end{abstract}

Seit der Einführung von Polyacrylamidgel („PAA“-Gel) als Träger für Elektrophoresen durch RAYMOND und WeINTraub (1) hat sich dieses synthetische Gel als vielseitig verwendbar erwiesen (2). Die Trennung eines Eiweißgemisches in Polyacrylamidgel erfolgt wie in Stärke-

\footnotetext{
Abkürzungen: PAA-Gel = Polyacrylamid-Gel DMAPN $=$ Dimethylaminopropionitril AP $=$ Ammoniumpersulfat AC $=$ Acrylamid MBAC $=\mathrm{N}, \mathrm{N}^{\prime}$-Methylenbisacrylamid
}

gel nicht nur nach verschiedener Ladung, sondern auch nach dem Molekulargewicht, bzw. der Molekülkonfiguration der Komponenten, doch ist es im Gegensatz $z \mathfrak{u}$ Stärkegel einfacher und stets reproduzierbar darstellbar und gegen physikalische und chemische Einflüsse widerstandsfähiger.

Bei der Elektrophorese in PAA-Gel ist es üblich, in vertikal gelagerten Gelen zu arbeiten $(3,4,5)$. Eine Verzerrung der Zonen durch Erwärmung oder durch Austrocknung des Gels während des Elektrophoreselaufes 